\title{
Pericyte-derived extracellular vesicles-mimetic nanovesicles improves peripheral nerve regeneration in mouse models of sciatic nerve transection
}

\author{
GUO NAN YIN ${ }^{1 *}$, TAE YOUNG SHIN ${ }^{2 *}$, JIYEON OCK ${ }^{1 *}$, MIN-JI CHOI ${ }^{1}$, ANITA LIMANJAYA $^{1}$, MI-HYE KWON ${ }^{1}$, \\ FANG-YUAN LIU ${ }^{1}$, SOON-SUN HONG ${ }^{3}$, JU-HEE KANG ${ }^{4}$, YONG SONG GHO ${ }^{5}$, JUN-KYU SUH ${ }^{1}$ and JI-KAN RYU ${ }^{1}$ \\ ${ }^{1}$ Department of Urology and National Research Center for Sexual Medicine, Inha University School of Medicine, \\ Incheon 22332; ${ }^{2}$ Department of Urology, Ewha Woman's University School of Medicine, Seoul 07804; \\ ${ }^{3}$ Department of Biomedical Sciences, College of Medicine, Program in Biomedical Science and Engineering, Inha University; \\ ${ }^{4}$ Department of Pharmacology and Medicinal Toxicology Research Center, \\ Inha University College of Medicine, Incheon 22332; ${ }^{5}$ Department of Life Sciences, \\ Pohang University of Science and Technology, Pohang, Gyeongsangbuk-do 37673, Republic of Korea
}

Received September 30, 2021; Accepted December 2, 2021

DOI: $10.3892 / \mathrm{ijmm} .2021 .5073$

\begin{abstract}
Pericyte-derived extracellular vesicle-mimetic nanovesicles (PC-NVs) play an important role in the improvement of erectile function after cavernous nerve injury. However, the impact of PC-NVs on the peripheral nervous system (PNS), such as the sciatic nerve, is unclear. In this study, PC-NVs were isolated from mouse cavernous pericytes (MCPs). A sciatic nerve transection (SNT) model was established using 8-week-old C57BL/6J mice. The sciatic nerve was harvested 5 and 14 days for immunofluorescence and western blot studies. Function studies were evaluated by performing the rotarod test and walking track analysis. The results demonstrated that PC-NVs could stimulate endothelial cells, increase neuronal cell content, and increase macrophage and Schwann cell presence at the proximal stump rather than the distal stump in the SNT model, thereby improving angiogenesis and nerve regeneration in the early stage of sciatic nerve regeneration. In addition, PC-NVs also increased the expression of neurotrophic factors (brain-derived nerve growth factor, neurotrophin-3 and nerve growth factor) and the activity of the cell survival signaling pathway (PI3K/Akt signaling), and reduced the activity of the JNK signaling
\end{abstract}

Correspondence to: Professor Jun-Kyu Suh or Professor Ji-Kan Ryu, Department of Urology and National Research Center for Sexual Medicine, Inha University School of Medicine, 7-206 3rd Street, Shinheung-Dong, Jung-Gu, Incheon 22332, Republic of Korea

E-mail: jksuh@inha.ac.kr

E-mail: rjk0929@inha.ac.kr

*Contributed equally

Key words: angiogenesis, pericytes, endothelial cell, extracellular vesicle, macrophage, nanovesicle, neurovascular regeneration, neurotrophic factor, peripheral nervous system, sciatic nerve transection pathway. Additionally, after 8 weeks of local application of PC-NVs in SNT model mice, their motor and sensory functions were significantly improved, as assessed by performing the rotarod test and walking track analysis. In conclusion, the present study showed that the significant improvement of neurovascular regeneration in mice following treatment with PC-NVs may provide a favorable strategy for promoting motor and sensory regeneration and functional recovery of the PNS.

\section{Introduction}

Peripheral nerve injury causes a loss of sensory and motor function, and has a significant impact on the quality of life (1). In the United States alone, $>200,000$ nerve repair treatments are performed each year (2). For short lengths of nerve damage, the conventional clinical procedure, end-to-end anastomosis, is appropriate (3). If the damaged nerve is too long to be repaired with tension-free sutures, autologous transplantation can be used for nerve reconstruction, which is considered the 'gold standard' of contemporary nerve repair treatment (4). In recent decades, several configurations of potential autografts for nerve conduits have been investigated (5). Despite recent advances in the understanding of nerve regeneration and surgical techniques, complete functional repair of damaged nerves is rarely achieved (6).

Pericytes, which promote vessel growth and maintain the neurovascular unit, are thought to be progenitor cells and may play a role in neurovascular regeneration (7). It has also been proposed that peripheral nerve pericytes may stimulate axon regeneration and barrier enhancement, preventing neuronal loss in the central nervous system $(8,9)$. Degeneration of pericytes has been observed in diseases related to neurovascular dysfunction, such as dementia and Alzheimer's disease (10). Our previous study showed that pericytes enhanced neurovascular regeneration in a mouse model of cavernous nerve injury (11).

Several researchers have demonstrated that extracellular vesicles (EVs) incorporate proteins, lipids, and RNA, which 
are involved in physiological and pathological communications between cells $(12,13)$. Previous studies have shown that EVs from particular cell types and environments can affect tissue regeneration $(14,15)$. However, one of the most significant disadvantages of EVs is their poor production yield (16). Therefore, to overcome this limitation, a mini extruder system was developed, and >100-fold greater EV-mimetic NVs were extracted from different cell types, such as embryonic stem cells (ESCs) and mouse cavernous pericytes (MCPs). In addition, ESC-derived EV-mimetic NVs (ESC-NVs) and MCP-derived EV-mimetic NVs (PC-NVs) showed similar qualities to natural EVs, thus might be helpful for studies on neurovascular regeneration $(17,18)$. However, the detailed molecular mechanisms by which PC-NVs promote nerve regeneration remains largely unknown.

The sciatic nerve is the longest nerve in the human body. It is composed of motor and sensory fibers, and has been used as a model of neurovascular regeneration. Regeneration of severed nerves requires polarized vasculature induced by macrophages in the hypoxic bridge to guide the collective migration of Schwann cells (19). Our recent studies showed that ESC-NVs could rescue erectile function in diabetic mice, whereas PC-NVs significantly improved erectile dysfunction in a mouse cavernous nerve injury (CNI) model $(17,18)$. However, there are no studies assessing the functional evaluation of PC-NVs in the peripheral nervous system (PNS), to the best of our knowledge. Therefore, it was hypothesized that PC-NVs may also induce peripheral nervous regeneration through these aforementioned effects, including macrophage-induced angiogenesis to guide Schwann cell migration.

The aim of the present study was to evaluate the role of PC-NVs in peripheral nerve regeneration. The results showed that the exogenous delivery of PC-NVs increased the content of endothelial cells, macrophages, Schwann cells and neuronal cells in the sciatic nerve transection (SNT) model, thereby inducing neurovascular regeneration. Therefore, we hypothesize that PC-NVs may display a protective effect and accelerate nerve recovery, thereby improving damaged nerve function.

\section{Materials and methods}

Ethics statement and animal study design. In total, 85 adult male C57BL/6J mice (8 weeks old; weight, 20-25 g; Orient Bio, Inc.) were used in the present study: 10 for the MCP primary culture and $\mathrm{PC}-\mathrm{NV}$ isolation, 45 for mouse sciatic nerve neurovascular regeneration and pathway signaling experiments, and 30 for the motor function recovery test. All animals' health and behavior were monitored every day and experiments performed in this study were approved by the Institutional Animal Care and Use Committee of Inha University (approval no. 171129-527). Mice were fed with commercial standard laboratory food and water ad libitum, and maintained at room temperature $\left(23 \pm 2^{\circ} \mathrm{C}\right)$ with $40-60 \%$ humidity, specific pathogen-free conditions and 12-h light/dark cycles. The cage, bedding, food and water were strictly disinfected. All animals were placed under general anesthesia with intramuscular injections of ketamine $(100 \mathrm{mg} / \mathrm{kg})$ and xylazine $(5 \mathrm{mg} / \mathrm{kg})$ before preparing the models. All animals were euthanized by $100 \% \mathrm{CO}_{2}$ gas replacement rate at $10-30 \%$ container volume/min, and cessation of the heartbeat and respiratory arrest were confirmed before harvesting tissues. No mice were found dead during any of the experimental procedures

$M C P$ culture, preparation and characterization of $P C-N V s$. The MCP primary cultures were performed as described previously (20). Briefly, 8-week-old male C57BL/6J mice were euthanized immediately, the urethra and dorsal neurovascular bundle were removed from the penis tissue and only the corpus cavernosum tissues were used. The corpus cavernosum tissues were sectioned into 1-2 mm sections and cultured at $37^{\circ} \mathrm{C}$ with $5 \% \mathrm{CO}_{2}$ in DMEM (Gibco; Thermo Fisher Scientific, Inc.) supplemented with $20 \%$ FBS (Gibco; Thermo Fisher Scientific, Inc.), $1 \%$ penicillin/streptomycin (Gibco; Thermo Fisher Scientific, Inc.) and $10 \mathrm{nM}$ human pigment epithelium-derived factor (Sigma-Aldrich; Merck KGaA). The medium was changed every 2 days, and the cells were sub-cultured after 10 days. Cells from passages 2-4 were used for all of the experiments.

As described in our previous study, a mini extruder system (Avanti Polar Lipids, Inc.) was used to prepare PC-NVs (17). Briefly, MCPs were detached with $0.25 \%$ trypsin-EDTA (Invitrogen; Thermo Fisher Scientific, Inc.) and re-suspended in HEPES buffer solution (HBS; Gibco; Thermo Fisher Scientific, Inc.). The cell suspension was extruded 10 times through filters with 10,5 and $1 \mu$ m polycarbonate membranes (Nuclepore; Whatman plc; Cytiva). Next, they were subjected to ultracentrifugation at $100,000 \times \mathrm{g}$ for $2 \mathrm{~h}$ at $4^{\circ} \mathrm{C}$ with a step gradient, which was overlapped with $50 \%$ iodixanol $(1 \mathrm{ml}$; Axis-Shield Diagnostics, Ltd.) and 10\% iodixanol (2 ml) and the extruded samples were placed $(7 \mathrm{ml})$ on top. The PC-NVs were filtered through a $0.45 \mu \mathrm{m}$ filter (Altmann Analytik Gmbh \& Co. KG) and stored at $-80^{\circ} \mathrm{C}$ until subsequent analysis. The EXOCET exosome quantitation analysis kit (System Biosciences, LLC) was used to quantify the PC-NVs, and their concentration was adjusted to $1 \mu \mathrm{g} / \mu \mathrm{l}$.

The morphology of the PC-NVs was detected by transmission electron microscopy (TEM; Electron Microscopy Sciences) as described previously (17). The PC-NVs were verified by determining the expression of one negative and three positive NV markers by western blotting (according to the western blotting protocol): Golgi matrix protein 130 (GM130; negative marker; cat. no. 610822; 1:1,000; BD Biosciences), programmed cell death 6-interacting protein (Alix; positive marker; cat. no. NB100-65678; 1:1,000; Novus Biologicals, LLC), tumor susceptibility 101 (TSG101; positive marker; cat. no. NB200-112; 1:1,000, Novus Biologicals, LLC) and CD81 (cat. no. NBP1-77039; positive marker; 1:1,000; Novus Biologicals, LLC).

Fluorescence dye labelling of the PC-NVs for the tracking analysis. The PC-NVs were labelled with 1,1'-Dioctadecyl-3,3,3',3'-Tetramethylindodicarbocyanine, 4-Chlorobenzenesulfonate salt (DiD) red-fluorescent dye (Thermo Fisher Scientific, Inc.) according to the manufacturer's instructions. Briefly, $2.5 \mu \mathrm{l} \mathrm{DiD}$ dye solution was added to $100 \mu \mathrm{g}$ PC-NVs in a total volume of $500 \mu \mathrm{l} \mathrm{HBS}$, incubated at room temperature for $10 \mathrm{~min}$ and then further diluted with $9.5 \mathrm{ml}$ cold HBS. The samples were then ultracentrifuged at $100,000 \mathrm{x} \mathrm{g}$ for $2 \mathrm{~h}$ at $4^{\circ} \mathrm{C}$. Next, the PC-NVs pellet in HBS 
were centrifuged again at $100,000 \mathrm{xg}$ for $2 \mathrm{~h}$ at $4^{\circ} \mathrm{C}$ to remove the free DiD dye. The particles containing the DiD-labeled PC-NVs were then resuspended in HBS and used for tracking analysis.

Establishment of the sciatic nerve crushing (SNC) and SNT model. In order to evaluate the neurovascular regenerative ability of the PC-NVs, the SNC and SNT model were prepared as described previously $(21,22)$. The SNC model was used for the tracking analysis of the injected PC-NVs. The 8-week-old male C57BL/6J mice were immediately placed under general anesthesia with intramuscular injections of ketamine $(100 \mathrm{mg} / \mathrm{kg})$ and xylazine $(5 \mathrm{mg} / \mathrm{kg})$. The right hind paw sciatic nerves were exposed and crushed with a non-serrated needle holder under uniform pressure for $30 \mathrm{sec}$. After suturing the muscle layer, DiD-labeled PC-NVs $(5 \mu \mathrm{g} / 100 \mu \mathrm{l})$ were immediately injected around the crushed sciatic nerve, and the skin layer was sutured. A total of $24 \mathrm{~h}$ after SNC model preparation, the mice from all groups were euthanized and the nerves were harvested after the confirming a lack of heartbeat and respiratory arrest. The nerves were fixed in $4 \%$ paraformaldehyde overnight at $4{ }^{\circ} \mathrm{C}$. After fixing, a longitudinal section $(15 \mu \mathrm{m})$ was prepared and the DiD-labeled PC-NVs were observed under a confocal fluorescence microscope (K1-Fluo; Nanoscope Systems, Inc.).

The SNT model was used to study the sciatic nerve function index, as well for immunofluorescence and western blot experiments. The surgical procedure was the same as that of the SNC model. The 8-week-old male C57BL/6J mice were immediately anesthetized, and the right hind paw sciatic nerve was exposed and cut across the middle of the thigh with scissors. After suturing the muscle layer, HBS or PC-NVs (1 or $5 \mu \mathrm{g}$, respectively) was immediately injected around the SNT and the skin layer was sutured. A total of 5 and 14 days after SNT model preparation, the mice from all of the groups were euthanized, and the nerves were harvested after confirmation of a lack of heartbeat and respiratory arrest. The nerves were fixed in $4 \%$ paraformaldehyde overnight at $4^{\circ} \mathrm{C}$ and a longitudinal section $(15 \mu \mathrm{m})$ was prepared for subsequent experiments. A phase contrast image of the sciatic nerves was observed under a confocal fluorescence microscope.

Histological examination. For fluorescence microscopy, the sciatic nerve tissues were fixed in $4 \%$ paraformaldehyde for $24 \mathrm{~h}$ at $4^{\circ} \mathrm{C}$. After blocking with $1 \%$ BSA (cat. no. A3294-50G; Sigma-Aldrich; Merck KGaA) for $1 \mathrm{~h}$ at room temperature, the frozen tissue sections $(15 \mu \mathrm{m})$ were incubated with antibodies against CD31 (1:50; cat. no. MAB1398Z; MilliporeSigma), neurofilaments (NF; 1:100; cat. no. N-5389; Sigma-Aldrich; Merck KGaA), glial fibrillary acidic protein (GFAP; 1:100; cat. no.ab53554; Abcam),ionized calcium-binding adapter molecule 1 (Iba-1; 1:100; cat. no. ab5076; Abcam) and neural specific 10 (SCG10; 1:100; cat. no. NBP1-49461; Novus Biologicals, LLC) at $4^{\circ} \mathrm{C}$ overnight. After several washes with PBS, the sections were incubated with secondary antibodies against FITC Affinipure Goat anti-armenian hamster IgG $(\mathrm{H}+\mathrm{L})(1: 100$; cat. no. 127-095-160; Jackson ImmunoResearch Laboratories, Inc.), rhodamine (TRITC) Affinipure Rabbit anti-mouse IgG $(\mathrm{H}+\mathrm{L})$ (1:100; cat. no. 315-025-003; Jackson ImmunoResearch Laboratories, Inc.), Alexa Fluor 488 affinipure Donkey anti-Goat IgG (H+L) (1:100; cat. no. 705-545-147; Jackson
ImmunoResearch Laboratories, Inc.), Donkey anti-Rabbit IgG H\&L (DyLight ${ }^{\circledR}$ 550) (1:100; cat. no. ab98489; Abcam) for $2 \mathrm{~h}$ at room temperature. After washing with PBS, the samples were mounted in a solution containing DAPI (100 $\mu \mathrm{l}$; cat. no. H-1500; Vector Laboratories, Inc.). DAPI was used for nuclei labeling for $2 \mathrm{~min}$ at room temperature. The signals were visualized and digital images were obtained using a confocal fluorescence microscope (K1-Fluo; Nanoscope Systems, Inc.). Quantitative analysis of the histological examinations was performed using ImageJ version 1.34 (National Institutes of Health).

Rotarod test. The rotarod test was performed on a rotarod machine with automatic timers and falling sensors (cat. no. JD-A-07TS; Jeung-Do Bio \& Plant Co., Ltd.) as described previously (23). The 8-week-old male C57BL/6J mice were allowed to acclimate to the test room for $30 \mathrm{~min}$. The mice were trained on the rotarod for $5 \mathrm{~min}$ for 3 consecutive days under the following conditions: Initially set the rod to a low speed of $4 \mathrm{rpm}$, and then increased to $12 \mathrm{rpm}$ in $1 \mathrm{~min}$. A total of 3 days later, SNT model preparation and PC-NV injections were performed. The rotarod test was performed from 2 weeks after SNT model preparation under the following conditions: The rotarod was set to accelerate mode at a speed of 4-50 rpm for a maximum of $5 \mathrm{~min}$. The machine automatically recorded the time and latency of each test mouse before it fell. The longest falling latency of three test trials was selected to represent the motor function of each mouse. Rotarod test results were recorded at 2, 4, 6 and 8 weeks after the sciatic nerve injury. At 2 weeks after the final rotarod test, the mice from all of the groups were euthanized.

Walking track analysis. Motor function recovery was evaluated by calculating the sciatic nerve function index (SFI). Walking track analysis was carried out by using open narrow corridors, and the assessment of gait in this model has been widely used as previously described (24). The walking track analysis was performed immediately after the rotarod test (2, 4, 6 and 8 weeks after the sciatic nerve injury). Briefly, mouse footprints were obtained by painting the hind paws and then letting the mouse walk along a $5 \times 50 \mathrm{~cm}$ narrow corridor covered with paper. Based on previous a study (25), the tracks were evaluated according to three different parameters: i) Toe spread (TS), the distance between the first toe and the fifth toes; ii) intermediate toe spread (IT), the distance between the second, third and fourth toes; and iv) print length (PL), the distance between the third toe and the hind pad. The measurements were taken on both the experimental (E) and normal control side (N). SFI was calculated using the following formula: $-38.3[($ EPL-NPL)/NPL]+109.5[(ETSNTS)/NTS]+13.3[(EIT-NIT)/NIT]-8.8 (26). An SFI value close to 0 means normal nerve function, and a value close to -100 means complete dysfunction. All tracks were analyzed by an experimenter blinded to the group assignments. Pawprints were recorded at 2, 4, 6 and 8 weeks after the sciatic nerve injury. At 2 weeks after the final pawprint recordings, the mice from all of the groups were euthanized.

Western blot. The sciatic nerve tissues (from the cut site and at the area located $5 \mathrm{~mm}$ distal or proximal to the cut site) 
were harvested for western blot analysis $(n=4)$. The sciatic nerve tissues were lysed in RIPA buffer (Sigma-Aldrich; Merck KGaA) supplemented with protease inhibitors (GenDEPOT, LLC) and phosphatase inhibitors (GenDEPOT, LLC). Equal amounts of protein (30 $\mu \mathrm{g}$ per lane) were subjected $8-15 \%$ SDS-PAGE and then transferred to PVDF membranes. After blocking with 5\% non-fat dry milk for $1.5 \mathrm{~h}$ at room temperature, the membranes were incubated at room temperature with antibodies against brain-derived nerve growth factor (BDNF; 1:500; cat. no. sc-546; Santa Cruz Biotechnology, Inc.), nerve growth factor (NGF; 1:500; cat. no. sc-548; Santa Cruz Biotechnology, Inc.), neurotrophin-3 (NT-3; 1:500; cat. no. sc-547; Santa Cruz Biotechnology, Inc.), phosphorylated (p)-PI3K (1:500; cat. no. 4228; Cell Signaling Technology, Inc.), total (t)-PI3K (1:1,000; cat. no. 4292; Cell Signaling Technology, Inc.), p-Akt (1:500; cat. no 9271; Cell Signaling Technology, Inc.), t-Akt (1:1,000; cat. no. 9272; Cell Signaling Technology, Inc.), p-c-JUN (1:500; cat. no. 9261; Cell Signaling Technology, Inc.), t-c-JUN (1:1,000; cat. no. 9165; Cell Signaling Technology, Inc.), p-SAPK/JNK (1:500; cat. no. 9251; Cell Signaling Technology, Inc.), t-JNK (1:1,000; cat. no. 9252; Cell Signaling Technology, Inc.) and $\beta$-actin (1:2,000; cat. no. ab16051; Abcam) for $2 \mathrm{~h}$. The membranes were washed three times for $10 \mathrm{~min}$ with PBST $(0.1 \%$ Tween-20) at room temperature. Subsequently, the membranes were incubated with goat anti-rabbit IgG H\&L (HRP) (1:1,000; cat. no. ab6721; Abcam) and goat anti-mouse IgG H\&L (HRP) $(1: 1,000$; cat. no. ab6789; Abcam) secondary antibodies for $2 \mathrm{~h}$ at room temperature. The signals were visualized using an ECL (Amersham Pharmacia Biotech, Inc.) detection system. The results were quantified by densitometry analysis with ImageJ.

Statistical analysis. The data are expressed as the mean \pm SEM of at least four independent experiments. Statistical analyses were performed using one-way ANOVA followed by Tukey's post hoc test (Figs. 1-8) or an unpaired Student's t-test (Fig. 9) in GraphPad Prism version 8 (GraphPad Software, Inc.). $\mathrm{P}<0.05$ was considered to indicate a statistically significant difference.

\section{Results}

$P C-N V$ measurements. PC-NVs were extracted from MCPs according to previous methods (17) and a schematic overview of the experimental procedure is shown in Fig. 1A. The PC-NVs exhibited a unique cup-shaped form, similar to the shape of endogenous EVs seen on TEM (Fig. 1B). The diameter of the purified PC-NVs was $\sim 30 \mathrm{~nm}$. As previous studies have shown, the NVs also expressed EV surface markers, such as Alix, TSG101 and CD81 (17,18). Therefore, the extracted PC-NVs were characterized by western blot analysis. The expression of EV-positive markers, such as Alix, TSG101 and CD81, was higher in PC-NVs than in the PC lysate. Conversely, the expression of the EV-negative marker, GM130, was lower in the PC-NVs than in the PC lysates (Fig. 1C).

$P C-N V$ s tracking in the SNC model. In order to determine the distribution of exogenously injected PC-NVs, DiD was used to label the PC-NVs, which were then injected into the SNC model mice. After 24 h, PC-NVs labelled with DiD were primarily detected in the crush site, and only partially detected in the proximal and distal areas (Fig. 2). This data indicated that PC-NVs were more preferably absorbed by injured tissues.

$P C-N V$ s promote neurovascular regeneration in the SNT model. Given that PC-NVs play critical roles in cavernous nerve injury induced erectile dysfunction (18), we hypothesized that the PC-NVs could also influence neurovascular regeneration in peripheral nerves, such as the sciatic nerve. In order to determine the effect of PC-NVs on sciatic nerve regeneration, SNT was performed and PC-NVs (1 and $5 \mu \mathrm{g})$ were injected after the transection surgery (Fig. 3A). At day 5, phase images of the sciatic nerve showed that the high-dose of PC-NVs $(5 \mu \mathrm{g})$ group transection region contained a notable influx of blood vessels compared with the HBS group. Interestingly, the number of blood vessels increased markedly at the proximal stump rather than the distal stump (Fig. 3B). At day 14, phase images of the sciatic nerves showed that, compared with the HBS group, the sciatic nerve link in the high-dose of PC-NVs $(5 \mu \mathrm{g})$ group appeared to be closer and stronger (Fig. 3C).

In addition, consistent with the sciatic nerve phase images, immunofluorescence staining of the sciatic nerve with CD31 (an endothelial cell marker) and NF (an axonal marker) revealed that the high-dose of PC-NVs significantly induced the endothelial cells and axonal content compared with the HBS group, which may have led to the sciatic nerve becoming more vascularized. A low dose of PC-NVs $(1 \mu \mathrm{g})$ injection showed a partial effect on sciatic nerve neurovascular regeneration (Fig. 4). At day 14, the CD31 and NF immunofluorescence staining showed that the blood vessels and axons succeeded in crossing the bridged part in the PC-NVs $(5 \mu \mathrm{g})$ group (Fig. 5). Taken together, these data showed that PC-NVs promoted neurovascular regeneration in the SNT model.

$P C-N V$ s promote macrophage and Schwann cell content in the SNT model mice. During the regeneration of the sciatic nerve, macrophages play an important role in inducing the polarized vasculature in the nerve bridge and in guiding the migration of Schwann cells during $(19,21)$. To determine whether PC-NVs could regulate the expression of macrophages to promote sciatic nerve regeneration, immunofluorescence staining of the sciatic nerve with Iba-1 (a macrophage marker) and GFAP (a Schwann cell marker) was performed. The results showed that the high-dose of PC-NVs significantly increased the presence of macrophages and Schwann cells compared with the HBS group. Injection of a low dose of PC-NVs only resulted in a significant increase in the presence of macrophages, but not Schwann cells. In addition, both Iba-1 and GFAP expression levels were high in the proximal transected site, but not the distal site in the high-dose PC-NVs treatment group (Fig. 6). These data are consistent with previous studies showing $(19,27)$ that the increased presence of macrophages may provide signals to direct the movement of Schwann cells to assist them in crossing the gap left by the wound during the early stages of sciatic nerve regeneration.

$P C-N V$ s promote nerve regeneration in SNT model mice. SCG10 is expressed in adult sensory neurons and is an effective and selective marker for sensory axon regeneration during the early stages of axon regeneration (28). Therefore, in order 
A Schematic diagram of the isolation of PC-NVs

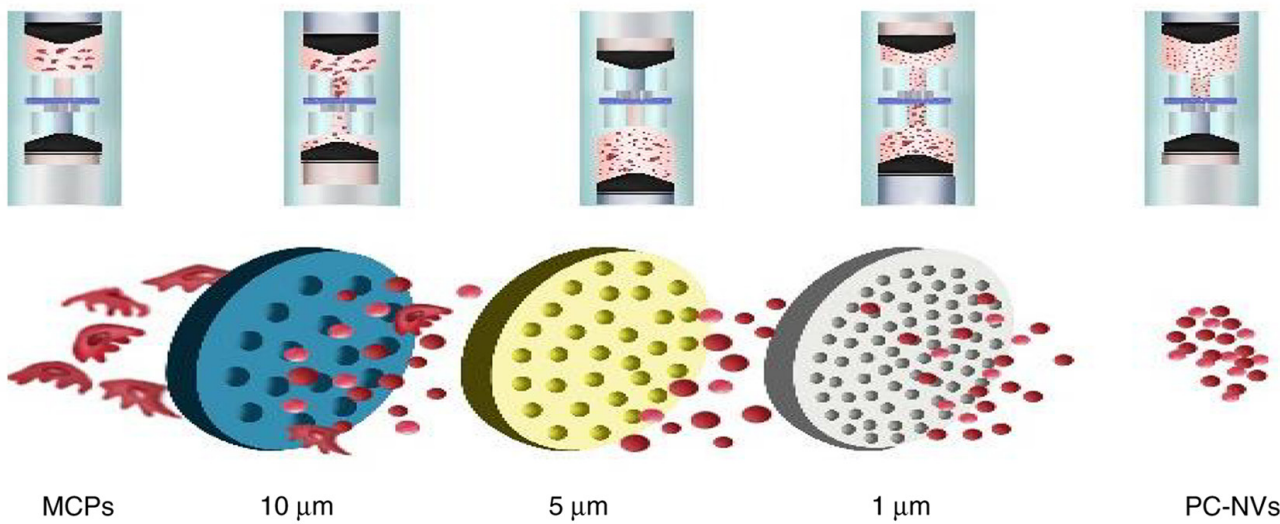

$\mathrm{B}$

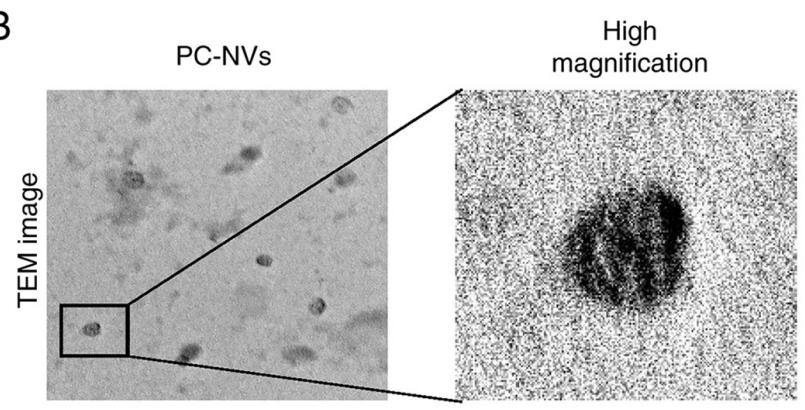

C
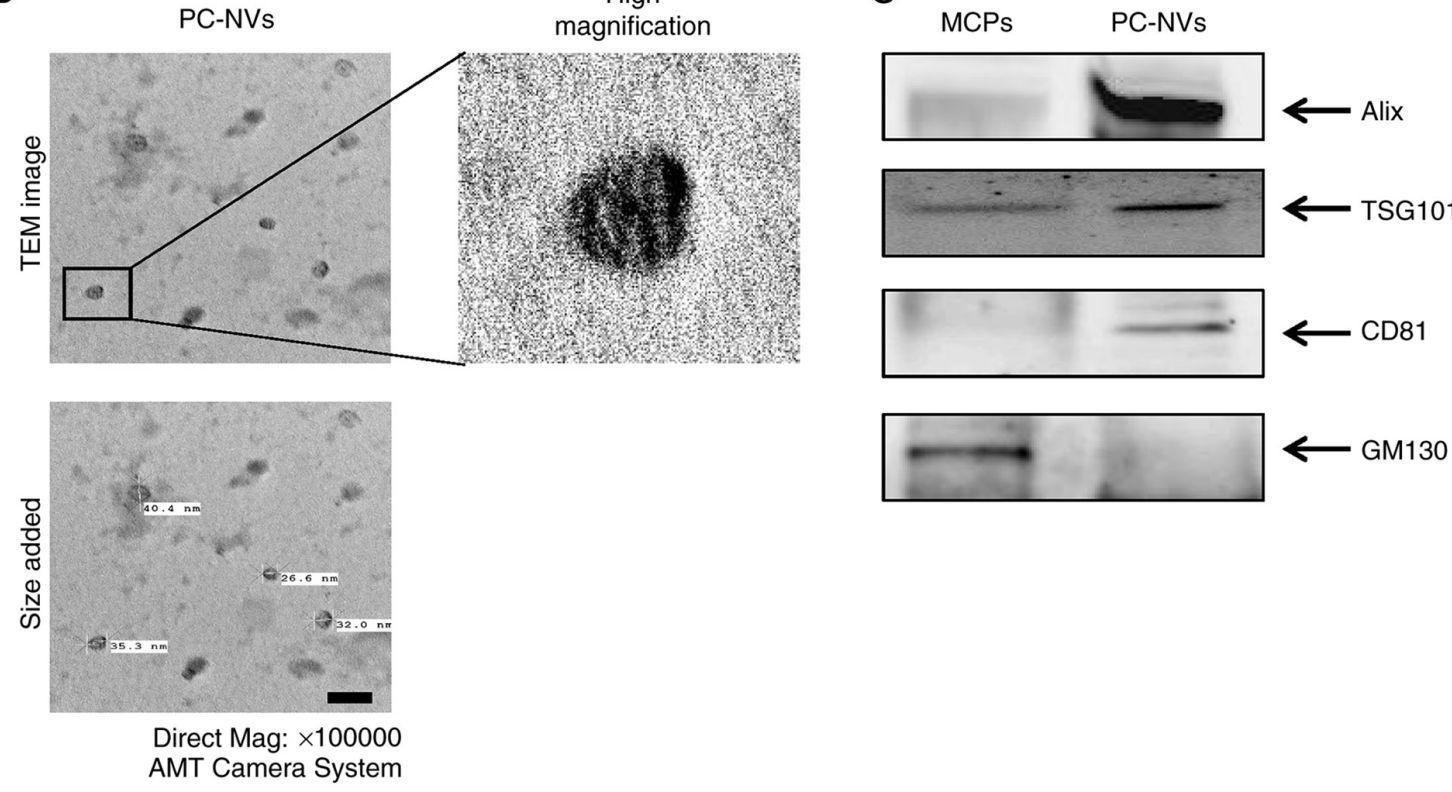

Figure 1. PC-NV isolation and characterization. (A) Schematic diagram of the isolation of PC-NVs. (B) Representative TEM phase images of PC-NVs Magnification, x100,000. (C) Representative western blots for EV-positive markers (Alix, TSG101 and CD81) and an EV-negative marker (GM130) in the MCP cell lysates and PC-NVs. EV, extracellular vesicle; MCP, mouse cavernous pericyte; PC-NV, pericyte-derived EV-mimetic nanovesicles; TEM, transmission electron micrograph; AMT, Advanced Microscopy Techniques; Alix, programmed cell death 6-interacting protein; TSG101, tumor susceptibility 101; GM130, golgi matrix protein 130 .

to evaluate the regenerative state in the early stage of axonal regeneration, immunofluorescence staining was performed for SCG10 in the SNT tissues after 5 days of treatment with PC-NVs. The results showed that, compared with the HBS group, low-dose and high-dose PC-NV treatment significantly increased the levels of SCG10 in the area of bridge (Fig. 7). This finding indicated that PC-NVs promoted nerve regeneration by increasing axon regeneration in the early stages of sciatic nerve regeneration.

$P C-N V s$ induced neurovascular regeneration by enhancing expression of NFs and survival signaling in SNT model mice. In the mammalian PNS, growth factor-mediated PI3K/Akt signaling is associated with intrinsic neurite outgrowth and synaptic plasticity (29). Consistent with previous reports $(18,29)$, it was found that PC-NVs also significantly upregulated the expression of NFs (BDNF, NT-3 and $\mathrm{NGF}$ ) and cell survival signaling-related proteins (PI3K/Akt signaling), whereas cell death signaling-related proteins, such as JNK, were significantly attenuated in the SNT model mice compared with those in the HBS group (Fig. 8). Collectively, these results suggested that regulation of BDNF, NT-3, NGF, PI3K, Akt, JNK and c-JUN occurred via PC-NV-mediated neurovascular regeneration in SNT model mice.

$P C-N V s$ promote the recovery of sciatic nerve function in SNT model mice. In order to evaluate the motor coordination, balance and sensory function recovery of the injured sciatic nerve, most researchers use the rotarod test and walking track analysis (23). Therefore, SNT was performed on mice locally treated with a high-dose of PC-NVs $(5 \mu \mathrm{g})$ around the transected site of the sciatic nerves and then made to undergo the rotarod test and walking track analysis every 2 weeks for a total of 8 weeks. The sensory motor coordination was evaluated by measuring the SFI. The results showed that the toe extension of SNT model mice was notably reduced compared 

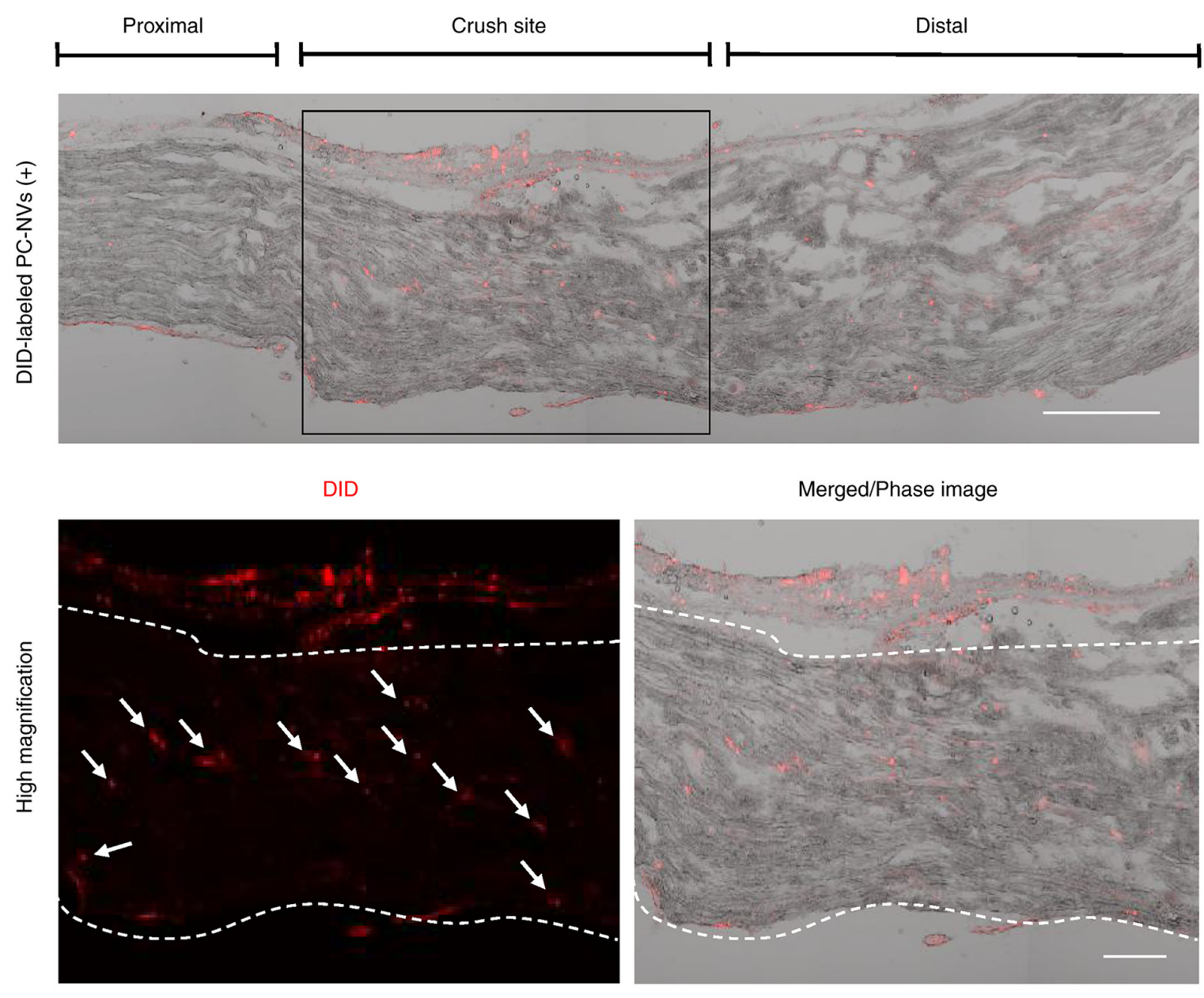

Sciatic nerve crushing (SNC) model

Figure 2. Tracking analysis of DiD-red fluorescently labeled PC-NVs in SNC model mice. DiD-labeled PC-NVs were injected around the crushed sciatic nerve for $24 \mathrm{~h}$. Representative phase images of mice sciatic nerve longitudinal sections showed that DiD-labeled PC-NVs were detected in the crushed site of the SNC model mice. White arrows indicate the absorbed PC-NVs. Scale bar in upper image, $200 \mu \mathrm{m}$. Scale bar in lower image, $100 \mu \mathrm{m}$. PC-NV, pericyte-derived extracellular vesicle-mimetic nanovesicle; SNC, sciatic nerve crushing; DiD, 1,1'-Dioctadecyl-3,3,3',3'-Tetramethylindodicarbocyanine, 4-Chlorobenzenesulfonate salt.

with that in the uncut group; however, after 8 weeks of local application of PC-NVs in SNT model mice, toe extension abilities were markedly improved (Fig. 9A). In the accelerating rotarod examination, local application of PC-NVs improved motor functions (latency and speed to fall) significantly from 4 weeks onwards compared with those in the transfected group (Fig. 9B and C). In addition, in the walking track analysis, walking patterns were analyzed to evaluate sensory and motor functionality during exercise. The pace of a normal mouse and an SNT mouse is shown in Fig. 9D. The SFI score was measured from 0 to 8 weeks after SNT. At 2 weeks after SNT, mice showed obvious neurological dysfunction (SFI score, $-69 \pm 2.35$ ). However, after PC-NV treatment, the SFI score improved significantly from 4 weeks (SFI score, $-44 \pm 2.58$ ) to 8 weeks (SFI score, $-28 \pm 4.98$ ) after SNT (Fig. 9E). Taken together, the functional examinations showed that the local application of PC-NVs significantly improved the impaired motor and sensory function in SNT model mice.

\section{Discussion}

Compared with the CNS, one of the conspicuous characteristics of the PNS is its greater regenerative ability (30). Although peripheral axons can regenerate and form functional connections, factors such as age, delay before intervention and type of injury determine the degree of functional recovery after repair $(31,32)$. Therefore, in order to improve regeneration, increasing the speed of axonal growth, increasing neuron survival and preventing neuronal apoptosis are potential strategies. In the present study, it was demonstrated that exogenous PC-NVs were capable of improving the injured sciatic nerve through the increased expression of NFs, promotion of the cell survival signaling pathway and attenuation of the apoptotic signaling pathway. Thus, determining the functions of PC-NVs and identifying its detailed mechanisms may provide important clues to assist in developing a rapid and effective treatment strategy for the repair of peripheral nerve damage with limited side effects.

Pericytes play a crucial role in the early phases of angiogenesis in damaged tissue, such as disintegration of the basement membrane and directing endothelial sprouting for angiogenesis (33). Previously, a relationship between peripheral nerve regeneration and angiogenesis following nerve damage was discovered $(11,19)$. Previous studies showed how various NVs, such as PC-NVs and ESC-derived EV-mimetic NVs (ESC-NVs), could restore the erectile function of the autonomic nervous 
A

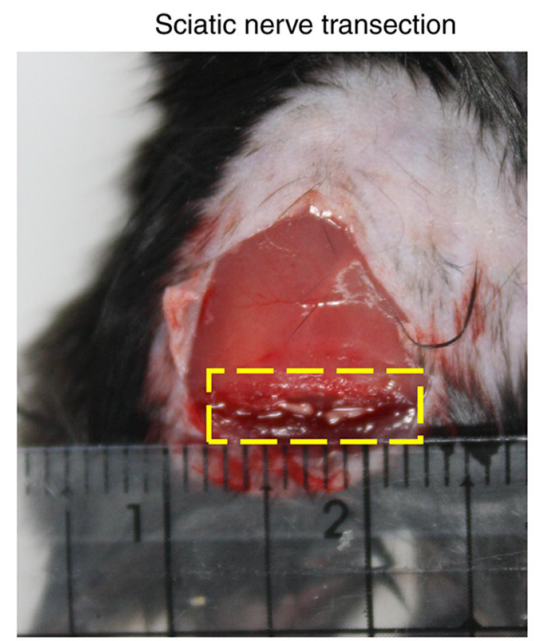

High magnification

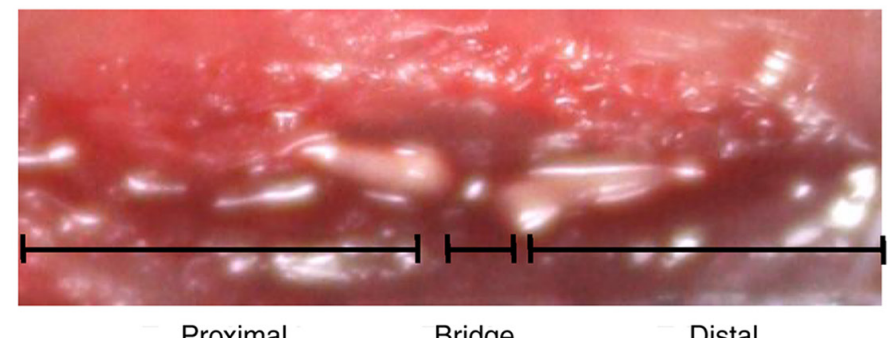

Bridge
Distal
B

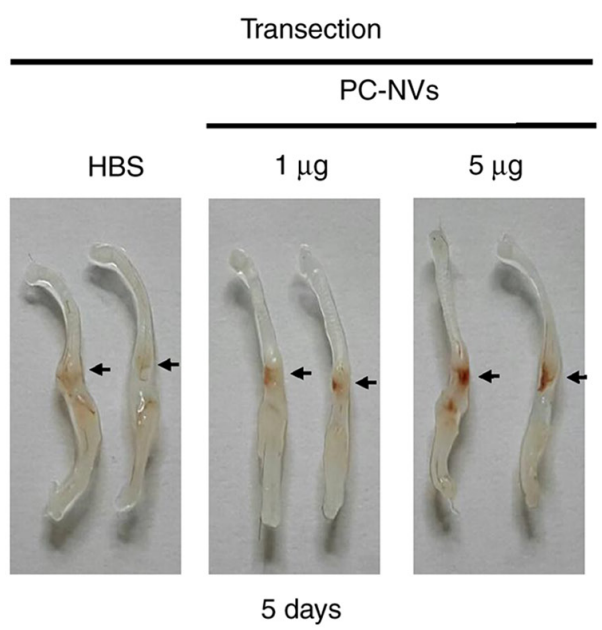

C

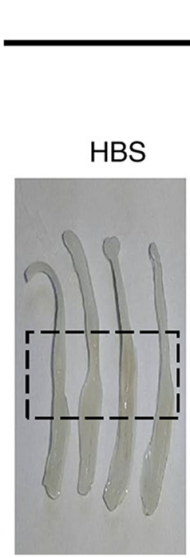

Transection

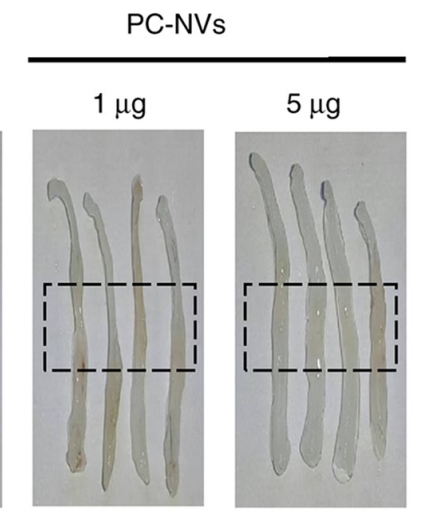

Proximal

14 days

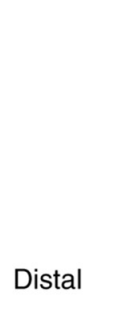

Figure 3. PC-NVs improve neurovascular regeneration in the SNT model. (A) Establishment of the SNT model in mice. After cutting the right hind paw sciatic nerves, a bridge of $\sim 1 \mathrm{~mm}$ was formed. The yellow dashed frame is magnified as the high-magnification image on the right. After suturing the muscle layer, HBS or PC-NVs (1 or $5 \mu \mathrm{g}$ ) were injected around the SNT site for (B) 5 and (C) 14 days. The arrow shows the proximal region of the bridge. The dotted box indicates the bridge part after transection from the proximal end to the distal end. PC-NV, pericyte-derived extracellular vesicle-mimetic nanovesicle; SNT, sciatic nerve transection; HBS, HEPES buffer solution.

system of diabetic mice $(17,18)$. Therefore, it was hypothesized that PC-NVs may play a beneficial role in the PNS.

To evaluate this hypothesis, PC-NVs were injected around the sciatic nerve immediately after transection. According to previous studies, the time point for sciatic nerve neurovascular regeneration is 5 days for angiogenesis and 14 days for nerve regeneration $(19,34)$. In the present study, high-dose PC-NVs $(5 \mu \mathrm{g})$ significantly promoted angiogenesis at the proximal stump in the transected sciatic nerve 5 days after treatment, and the sciatic nerve connections were closer and stronger in the PC-NVs $(5 \mu \mathrm{g})$ treatment group after 14 days of treatment. In addition, a previous study showed that macrophages may detect hypoxia within the neural bridge and generate a polarized vasculature, which provides a scaffold for Schwann cell migration (19). Schwann cells have been shown to migrate with blood vessels to damaged tissues, produce neurotrophic substances and use autophagy to clear the necrotic tissue produced by Wallerian degeneration, thereby promoting nerve regeneration (35). Consistently, the present study showed that PC-NVs increased the content of endothelial cells (CD31), macrophages (Iba-1) and Schwann cells (GFAP), and enhanced SCG10 expression in the transected sciatic nerve bridge. Based on these results, it was hypothesized that the Schwann cells induced by PC-NVs might be involved in the removal of necrotic tissues produced by Wallerian degeneration. However, at present, there are no relevant experimental results to support this hypothesis, thus more detailed experiments are required to assess. These findings strongly suggested that PC-NVs may serve an important role in peripheral nerve neurovascular regeneration.

It has been reported that Schwann cell-derived NVs could increase the production of NFs (BDNF, NT-3 and NGF), thereby improving neurite outgrowth in vitro (36). The activation of Akt signaling has been shown to promote cell proliferation and the migration of multiple cell types (37). In addition, previous studies have also shown that PC-NVs could improve erectile function by enhancing the expression of NFs and activating survival signaling in a mouse CNI model (18). Consistently, it was also found that a high-dose of PC-NVs significantly enhanced these factors.

Finally, in order to evaluate the role of PC-NVs in PNS functional regeneration, a rotarod test and walking track analysis were performed in the present study. The results showed that 
A Mouse sciatic nerve 5 days after transection

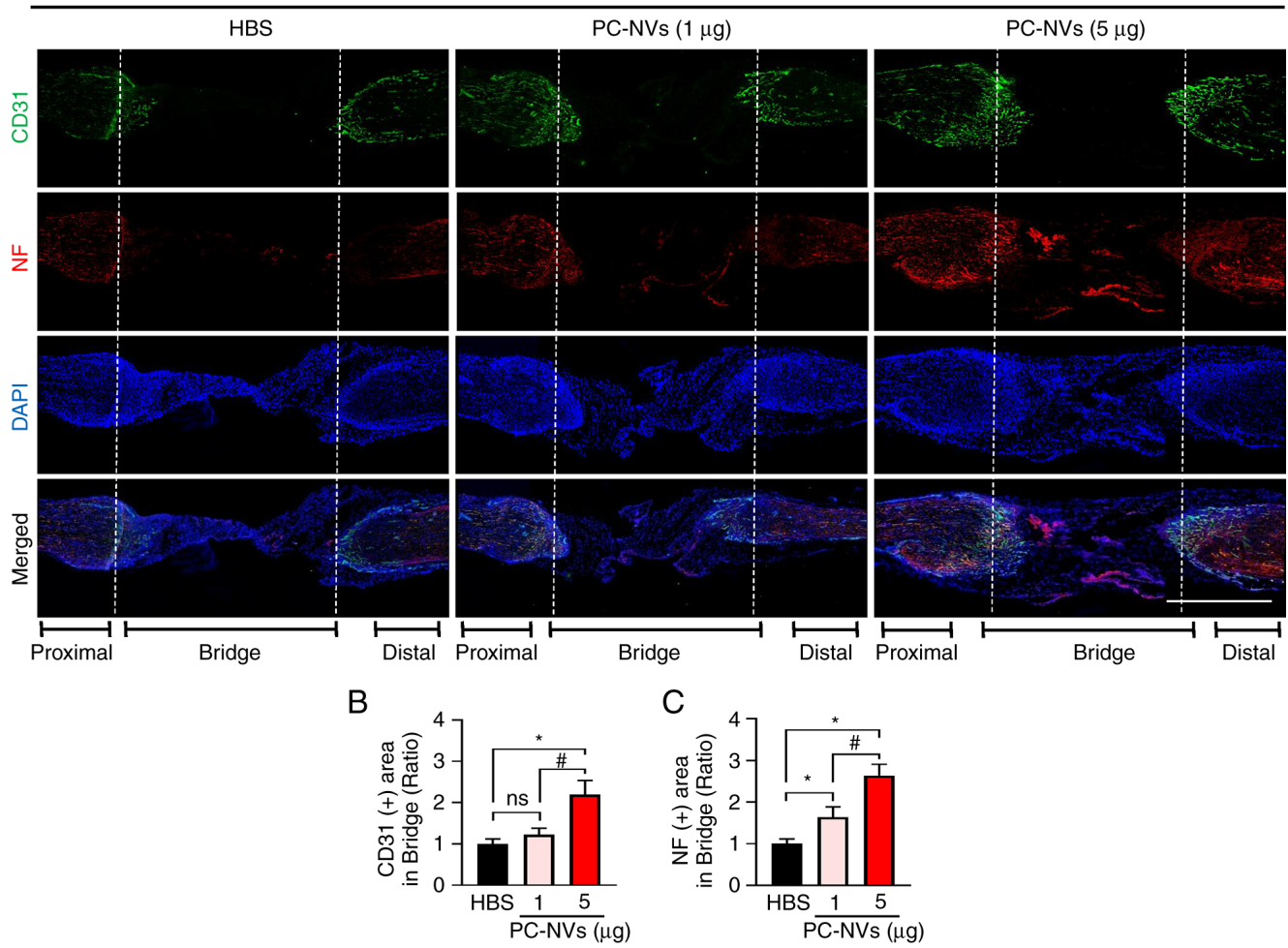

Figure 4. PC-NVs promote neurovascular regeneration 5 days after sciatic nerve transection. (A) Representative immunofluorescence staining (CD31 and NF) of transected sciatic nerves 5 days after injection with HBS or PC-NVs ( 1 and $5 \mu \mathrm{g})$. The sciatic nerve is divided into the proximal, bridge and distal regions by the dotted lines. Nuclei were labeled with the DAPI. Scale bar, $500 \mu \mathrm{m}$. Quantification of (B) CD31-expressing endothelial cells and (C) NF-expressing axonal content in the bridge. The results are presented as the mean $\pm \mathrm{SEM}$ of four repeats. The relative ratio of the HBS group was arbitrarily set to 1 . ${ }^{*} \mathrm{P}<0.001 \mathrm{vs}$. HBS group; ${ }^{\mathrm{P}} \mathrm{P}<0.001$ vs. PC-NV (1 $\mu \mathrm{g})$ group. ns, not significant; PC-NV, pericyte-derived extracellular vesicle-mimetic nanovesicle; HBS, HEPES buffer solution; NF, neurotrophic factor.

A

Mouse sciatic nerve 14 days after transection
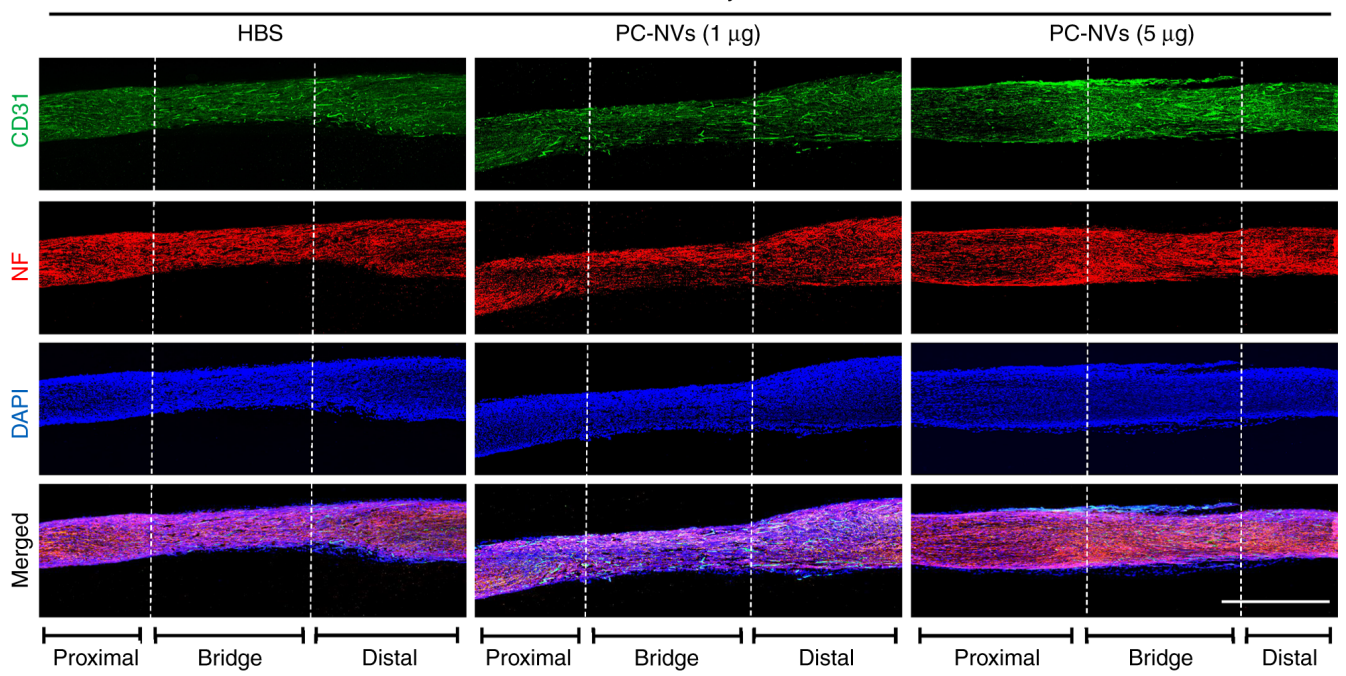

$\mathrm{B}$

C
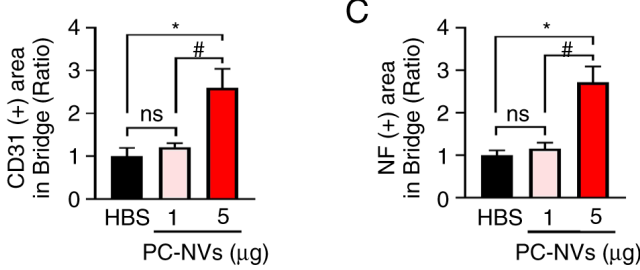

Figure 5. PC-NVs promote neurovascular regeneration 14 days after sciatic nerve transection. (A) CD31 and NF immunofluorescence staining of the transected sciatic nerve 14 days after injection with HBS and PC-NVs (1 and $5 \mu \mathrm{g}$ ). The sciatic nerve is divided into proximal, bridge and distal regions by the dotted lines. Nuclei were labeled with DAPI. Scale bar, $500 \mu \mathrm{m}$. Quantification of (B) CD31-expressing endothelial cells and (C) NF-expressing axonal content in the bridge. The results are presented as the mean \pm SEM of four repeats. The relative ratio of the HBS group was arbitrarily set to $1 .{ }^{*} \mathrm{P}<0.001$ vs. HBS group; ${ }^{\sharp} \mathrm{P}<0.001 \mathrm{vs}$. PC-NV (1 $\mu \mathrm{g})$ group. ns, not significant; PC-NV, pericyte-derived extracellular vesicle-mimetic nanovesicle; HBS, HEPES buffer solution; NF, neurotrophic factor. 
A Mouse sciatic nerve 5 days after transection
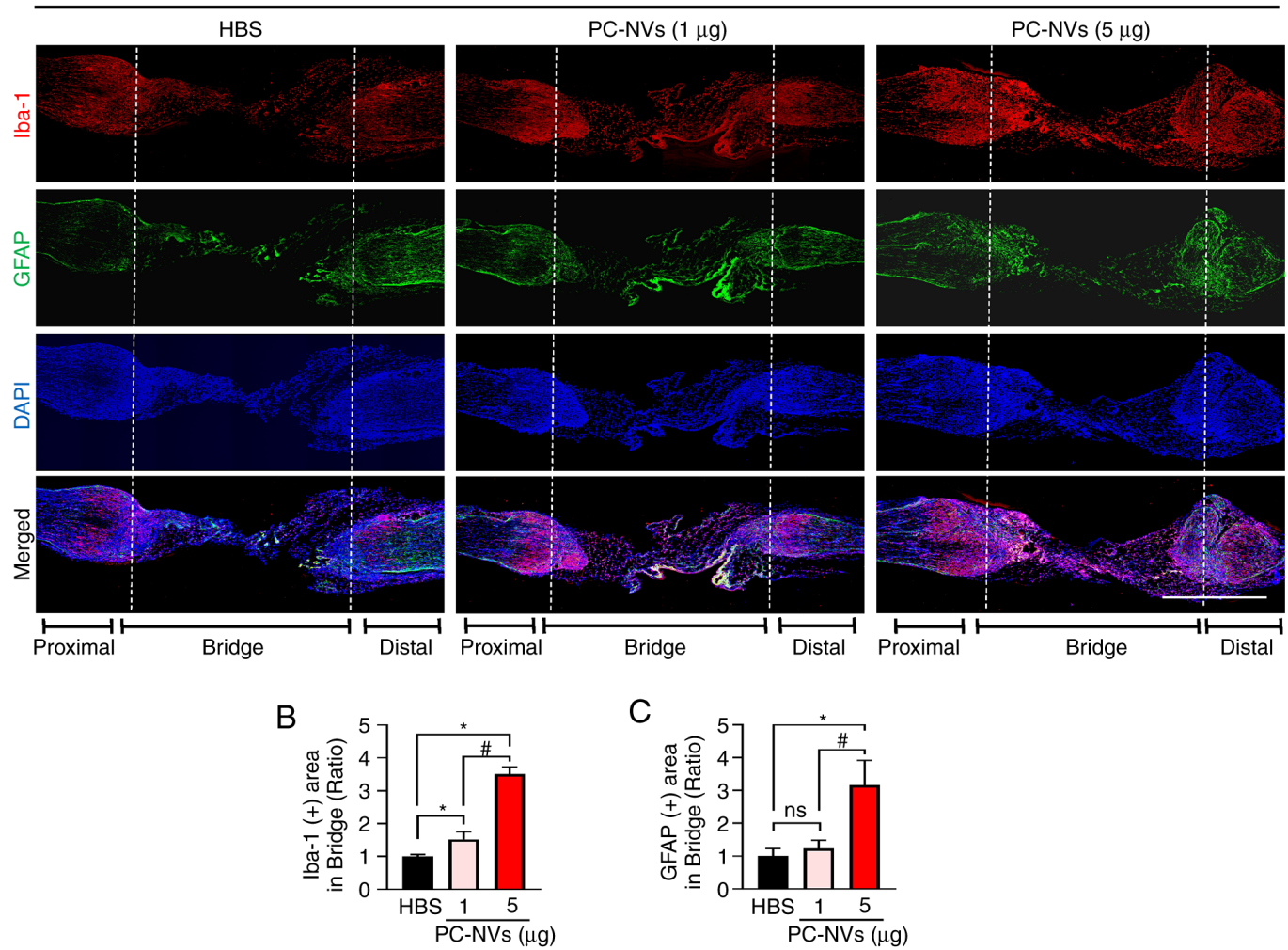

Figure 6. PC-NVs promote macrophage and Schwann cell presence 5 days after sciatic nerve transection. (A) Iba-1 (a macrophage marker) and GFAP (a Schwann cell marker) immunofluorescence staining in the transected sciatic nerve 5 days after injection with HBS and PC-NVs ( 1 and $5 \mu \mathrm{g})$. The sciatic nerve is divided into proximal, bridge and distal regions by the dotted lines. Nuclei were labeled with DAPI. Scale bar, $500 \mu \mathrm{m}$. Quantification of (B) Iba-1 expression in the macrophages and (C) GFAP expression in the Schwann cells in the bridge. The results are presented as the mean \pm SEM of four repeats. The relative ratio of the HBS group was arbitrarily set to $1 .{ }^{*} \mathrm{P}<0.001$ vs. HBS group; ${ }^{*} \mathrm{P}<0.001$ vs. $\mathrm{PC}-\mathrm{NV}(1 \mu \mathrm{g})$ group. ns, not significant; PC-NV, pericyte-derived extracellular vesicle-mimetic nanovesicle; HBS, HEPES buffer solution; GFAP, glial fibrillary acidic protein; Iba-1, ionized calcium-binding adapter molecule 1.

A

Mouse sciatic nerve 5 days after transection
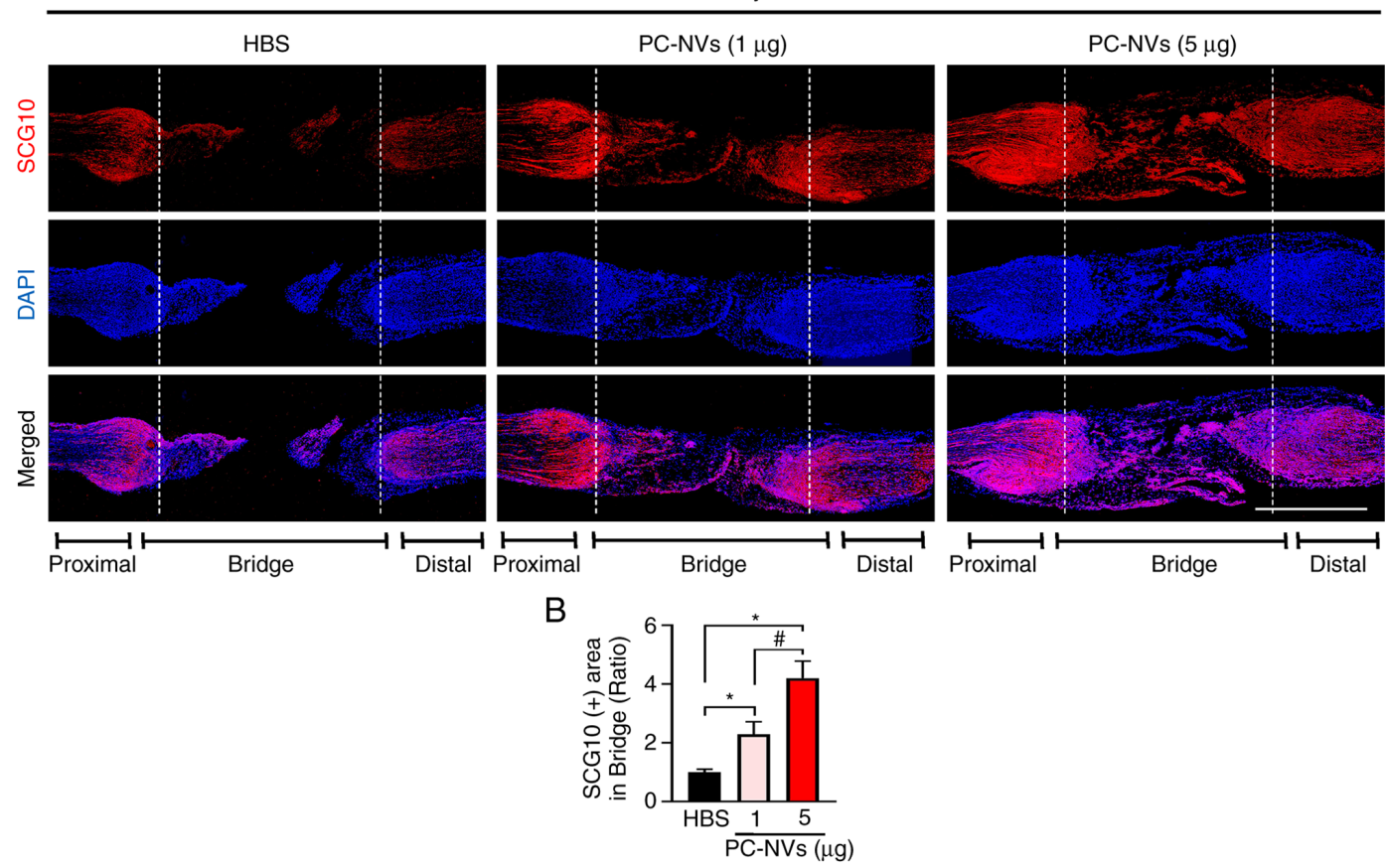

Figure 7. PC-NVs promote nerve regeneration 5 days after sciatic nerve transection. (A) SCG10 (a nerve regeneration marker) immunofluorescence staining in the transected sciatic nerve 5 days after injection with HBS and PC-NVs (1 and $5 \mu \mathrm{g})$. The sciatic nerve is divided into proximal, bridge and distal regions by the dotted lines. Nuclei were labeled with DAPI. Scale bar, $500 \mu \mathrm{m}$. (B) Quantification of SCG10 expression in the bridge. The results are presented as the mean \pm SEM of four repeats. The relative ratio of the HBS group was arbitrarily set to 1 . ${ }^{*} \mathrm{P}<0.001 \mathrm{vs}$. HBS group; ${ }^{*} \mathrm{P}<0.001 \mathrm{vs}$. $\mathrm{PC}-\mathrm{NV}(1 \mu \mathrm{g})$ group. PC-NV, pericyte-derived extracellular vesicle-mimetic nanovesicle; HBS, HEPES buffer solution; SCG10, ionized calcium-binding adapter molecule 1. 
A

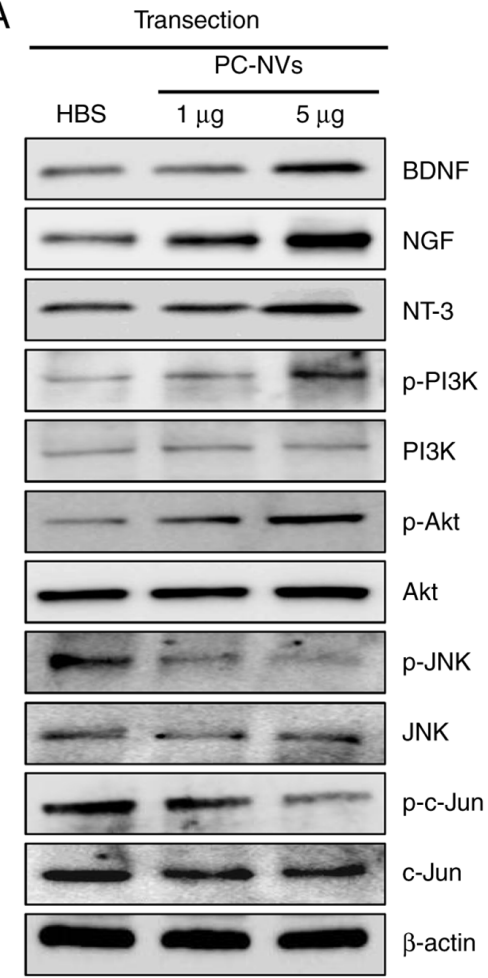

B

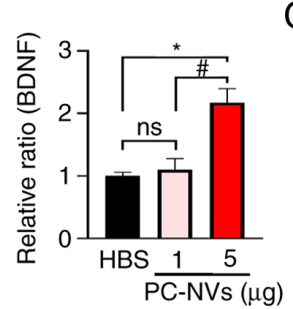

$E$

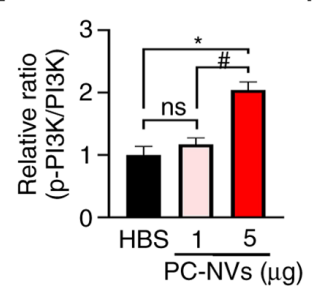

C

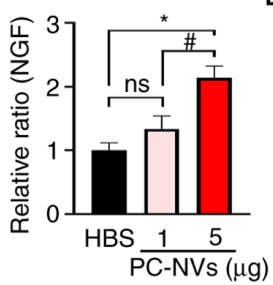

D

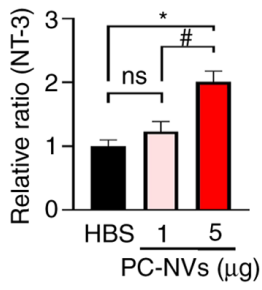

$\mathrm{F}$
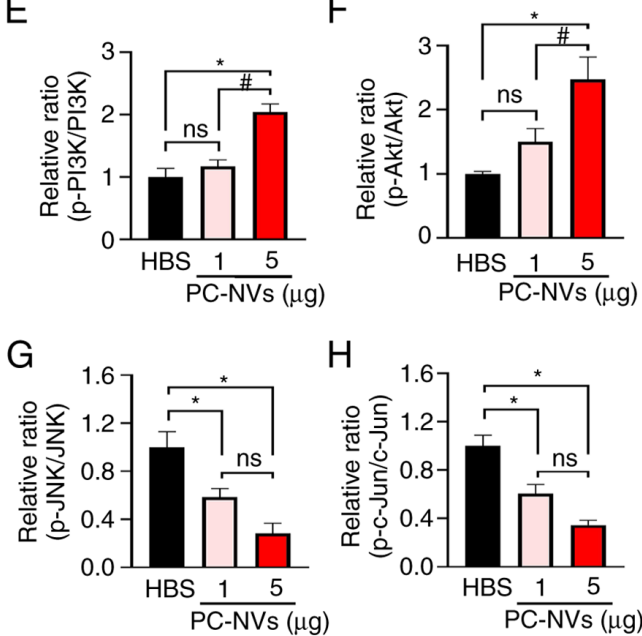

Figure 8. PC-NVs induce neurovascular regeneration by enhancing NF expression and survival signaling in SNT model mice. (A) Representative western blot for NFs (BDNF, NT-3 and NGF), survival signaling (activation of PI3K and Akt) and cell death signaling (activation of JNK and c-Jun) in sciatic nerve tissue from SNT model mice at 5 days after injection with HBS or PC-NVs (1 and $5 \mu \mathrm{g}$ ). Data are presented as the relative density of (B) BDNF, (C) NGF, (D) NT-3, (E) p-PI3K/PI3K, (F) p-Akt/Akt, (G) p-JNK/JNK and (H) p-c-Jun/c-Jun, with $\beta$-actin as the loading control. Each bar depicts the mean \pm SEM of four repeats. The relative ratio of the HBS group was arbitrarily set to $1 .{ }^{*} \mathrm{P}<0.05$ vs. HBS group; ${ }^{*} \mathrm{P}<0.05$ vs. PC-NVs $(1 \mu \mathrm{g})$ group. ns, not significant. HBS, HEPES buffer solution; PC-NV, pericyte-derived extracellular vesicle-mimetic nanovesicle; SNT, sciatic nerve transection; NF, neurotrophic factor; BDNF, brain-derived nerve growth factor; NT-3, neurotrophin-3; NGF, nerve growth factor.

A

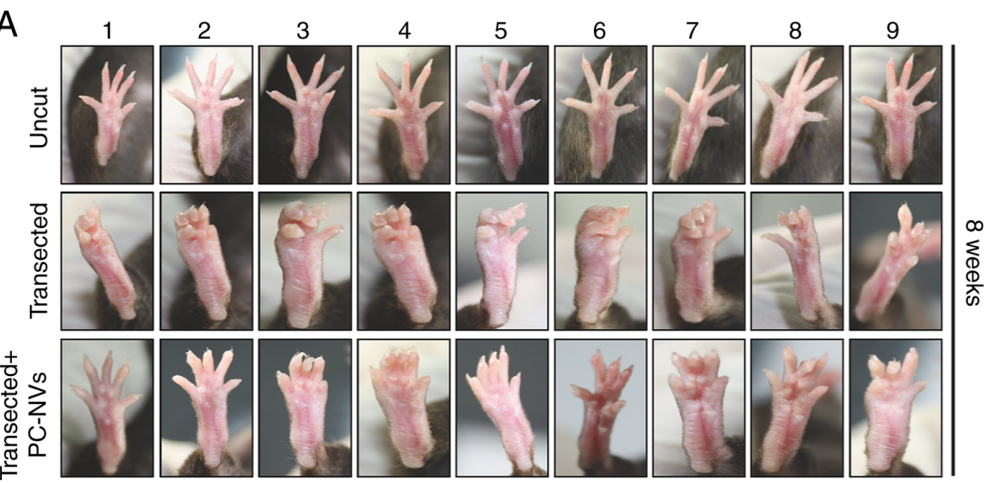

C

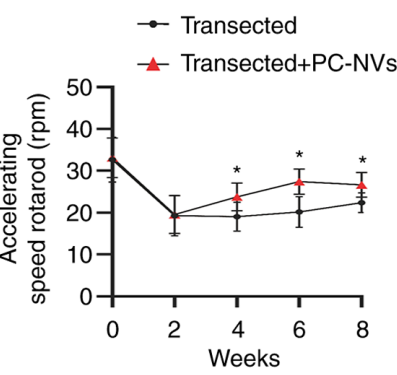

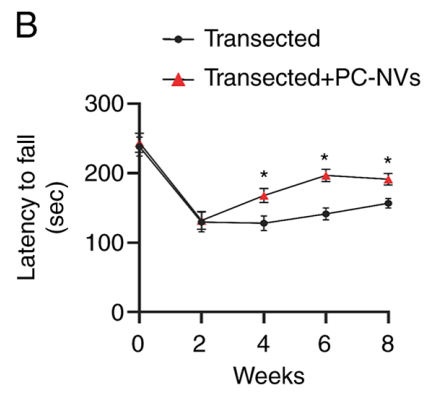

D

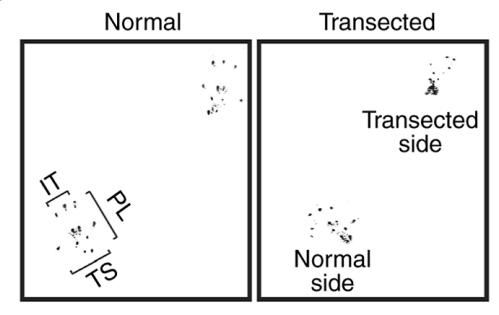

E

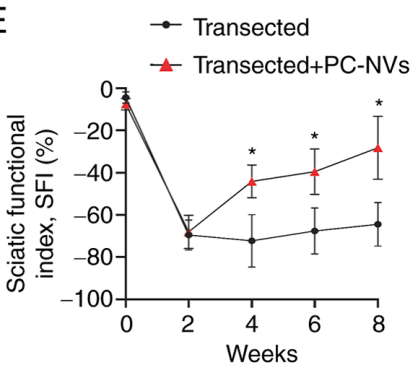

Figure 9. PC-NVs increase behavioral recovery and SFI in the SNT model. (A) Representative phase images of the mouse hind paw from each group after 8 weeks of treatment with PC-NVs. (B and C) Accelerating speed rotarod test in the SNT model mice treated with PC-NVs every 2 weeks for 8 weeks. Latency to fall and speed to fall were measured and the results are presented as the mean \pm SEM of nine repeats. (D) Representative normal and SNT model mice footprint tracks showing the measurements used to calculate the SFI. These include IT, TS and PL. (E) SFI assessments were conducted every 2 weeks for 8 weeks after the injection of PC-NVs in SNT model mice. The results are presented as the mean \pm SEM of nine repeats. "P<0.01 vs. transected groups. PC-NV, pericyte-derived extracellular vesicle-mimetic nanovesicle; SNT, sciatic nerve transection; SFI, sciatic function index; IT, intermediate toe spread; TS, toe spread; PL, print length. 
from 4 weeks after treatment, high-dose PC-NVs significantly improved the sensory-motor coordination and motor function. These findings indicated that local injection of PC-NVs may be a promising strategy for the treatment of peripheral nerve injury.

To the best of our knowledge, the present study was the first to evaluate the therapeutic impact of PC-NVs in sciatic nerve injury models. The current research did have several limitations. Only the effect of PC-NVs injected immediately after nerve injury was evaluated, and different time frames between injury and treatment should be assessed to determine the optimal time frame. Only male mice were used to prepare SNC and SNT models; therefore, the use of both female and male mice may be more meaningful for experimental study. The present study did elucidate which components of PC-NVs were involved in neurovascular regeneration and how long $\mathrm{PC}-\mathrm{NV}$ s remained at the injection site. Additional studies to evaluate which components of PC-NVs play a role in neurovascular regeneration may help in understanding the detailed mechanism of action of PC-NVs in neurovascular regeneration.

In summary, the present study showed that PC-NVs significantly enhanced cell survival signaling and the expression of NFs in neurovascular regeneration in the SNT mouse model. Thus, PC-NVs may serve as a novel treatment strategy for management of neurovascular diseases.

\section{Acknowledgements}

Not applicable.

\section{Funding}

This work was supported by the National Research Foundation of Korea (grant no. 2019R1A2C2002414), the Medical Research Center (grant no. NRF-2021R1A5A2031612), the Joint Grant [composed of the Korean government (Ministry of Science, ICT and Future Planning), the Bio \& Medical Technology Development Program of the National Research Foundation and the Korean government (MSIT); grant no. 2019069621] and the National Research Foundation of Korea (grant no. 2021R1A2C4002133).

\section{Availability of data and materials}

The datasets used and/or analyzed during the present study are available from the corresponding author on reasonable request.

\section{Authors' contributions}

GNY, TYS, JO, JKS and JKR conceived and designed the study. GNY, TYS, JO, MJC, AL, MHK, FYL, SSH, JHK and YSG performed the experiments, the data collection, statistical analysis, data interpretation. GNY, TYS, and JO confirm the authenticity of all the raw data. GNY, TYS, JO and JKR wrote the manuscript. All authors have read and approved the final manuscript.

\section{Ethics approval and consent to participate}

The experiments performed with animals were approved by the Institutional Animal Care and Use Committee of
Inha University (Incheon, Republic of Korea; approval no. 171129-527).

\section{Patient consent for publication}

Not applicable.

\section{Competing interests}

The authors declare that they have no competing interests.

\section{References}

1. Thomas S, Ajroud-Driss S, Dimachkie MM, Gibbons C, Freeman R, Simpson DM, Singleton JR, Smith AG, PNRR Study Group and Höke A: Peripheral neuropathy research registry: A prospective cohort. J Peripher Nerv Syst 24: 39-47, 2019.

2. Peters BR, Russo SA, West JM, Moore AM and Schulz SA: Targeted muscle reinnervation for the management of pain in the setting of major limb amputation. SAGE Open Med 8: 2050312120959180, 2020 .

3. Korus L, Ross DC, Doherty CD and Miller TA: Nerve transfers and neurotization in peripheral nerve injury, from surgery to rehabilitation. J Neurol Neurosurg Psychiatry 87: 188-197, 2016.

4. Hoshal SG, Solis RN and Bewley AF: Nerve grafts in head and neck reconstruction. Curr Opin Otolaryngol Head Neck Surg 28: 346-351, 2020.

5. Vijayavenkataraman S: Nerve guide conduits for peripheral nerve injury repair: A review on design, materials and fabrication methods. Acta Biomater 106: 54-69, 2020.

6. Al-Massri KF, Ahmed LA and El-Abhar HS: Mesenchymal stem cells in chemotherapy-induced peripheral neuropathy: A new challenging approach that requires further investigations. J Tissue Eng Regen Med 14: 108-122, 2020.

7. Geranmayeh MH, Rahbarghazi R and Farhoudi M: Targeting pericytes for neurovascular regeneration. Cell Commun Signal 17: 26, 2019.

8. Tamaki T,Hirata M, Nakajima N, Saito K, Hashimoto H, Soeda S, Uchiyama $Y$ and Watanabe M: A long-gap peripheral nerve injury therapy using human skeletal muscle-derived stem cells (Sk-SCs): An achievement of significant morphological, numerical and functional recovery. PLoS One 11: e0166639, 2016.

9. Rivera FJ, Hinrichsen B and Silva ME: Pericytes in multiple sclerosis. Adv Exp Med Biol 1147: 167-187, 2019.

10. Kisler K, Nelson AR, Rege SV, Ramanathan A, Wang Y, Ahuja A, Lazic D, Tsai PS, Zhao Z, Zhou Y, et al: Pericyte degeneration leads to neurovascular uncoupling and limits oxygen supply to brain. Nat Neurosci 20: 406-416, 2017.

11. Yin GN, Jin HR, Choi MJ, Limanjaya A, Ghatak K, Minh NN, Ock J, Kwon M, Song KM, Park HJ, et al: Pericyte-derived Dickkopf 2 regenerates damaged penile neurovasculature through an angiopoietin-1-Tie2 pathway. Diabetes 67: 1149-1161, 2018.

12. Li C, Qin F, Hu F, Xu H, Sun G, Han G, Wang T and Guo M: Characterization and selective incorporation of small non-coding RNAs in non-small cell lung cancer extracellular vesicles. Cell Biosci 8: 2, 2018.

13. O'Brien K, Breyne K, Ughetto S, Laurent LC and Breakefield XO: RNA delivery by extracellular vesicles in mammalian cells and its applications. Nat Rev Mol Cell Biol 21: 585-606, 2020.

14. Mäe MA, He L, Nordling S, Vazquez-Liebanas E, Nahar K, Jung B, Li X, Tan BC, Foo JC, Cazenave-Gassiot A, et al: Single-cell analysis of blood-brain barrier response to pericyte loss. Circ Res 128: e46-e62, 2021.

15. Liu Y and Holmes C: Tissue regeneration capacity of extracellular vesicles isolated from bone marrow-derived and adipose-derived mesenchymal stromal/stem cells. Front Cell Dev Biol 9: 648098, 2021.

16. Kim OY, Lee J and Gho YS: Extracellular vesicle mimetics: Novel alternatives to extracellular vesicle-based theranostics, drug delivery, and vaccines. Semin Cell Dev Biol 67: 74-82, 2017.

17. Kwon MH, Song KM, Limanjaya A, Choi MJ, Ghatak K, Nguyen NM, Ock J, Yin GN, Kang JH, Lee MR, et al: Embryonic stem cell-derived extracellular vesicle-mimetic nanovesicles rescue erectile function by enhancing penile neurovascular regeneration in the streptozotocin-induced diabetic mouse. Sci Rep 9: 20072, 2019. 
18. Yin GN, Park SH, Ock J, Choi MJ, Limanjaya A, Ghatak K, Song KM, Kwon MH, Kim DK, Gho YS, et al: Pericyte-derived extracellular vesicle-mimetic nanovesicles restore erectile function by enhancing neurovascular regeneration in a mouse model of cavernous nerve injury. J Sex Med 17: 2118-2128, 2020.

19. Cattin AL, Burden JJ, Van Emmenis L, Mackenzie FE, Hoving JJA, Calavia NG, Guo Y, McLaughlin M, Rosenberg LH, Quereda V, et al: Macrophage-induced blood vessels guide schwann cell-mediated regeneration of peripheral nerves. Cell 162: 1127-1139, 2015.

20. Yin GN, Park SH, Song KM, Limanjaya A, Ghatak K, Minh NN, Ock J, Ryu JK and Suh JK: Establishment of in vitro model of erectile dysfunction for the study of high-glucose-induced angiopathy and neuropathy. Andrology 5: 327-335, 2017.

21. Meng FW, Jing XN, Song GH, Jie LL and Shen FF: Prox 1 induces new lymphatic vessel formation and promotes nerve reconstruction in a mouse model of sciatic nerve crush injury. J Anat 237: 933-940, 2020

22. Chen B, Carr L and Dun XP: Dynamic expression of Slit1-3 and Robol-2 in the mouse peripheral nervous system after injury. Neural Regen Res 15: 948-958, 2020.

23. Lim EF, Nakanishi ST, Hoghooghi V, Eaton SE, Palmer AL, Frederick A, Stratton JA, Stykel MG, Whelan PJ, Zochodne DW, et al: AlphaB-crystallin regulates remyelination after peripheral nerve injury. Proc Natl Acad Sci USA 114: E1707-E1716, 2017.

24. Lee JI, Hur JM, You J and Lee DH: Functional recovery with histomorphometric analysis of nerves and muscles after combination treatment with erythropoietin and dexamethasone in acute peripheral nerve injury. PLoS One 15: e0238208, 2020.

25. KakaG,ArumJ,Sadraie SH,Emamgholi A and MohammadiA:Bone marrow stromal cells associated with poly L-Lactic-Co-Glycolic acid (PLGA) nanofiber scaffold improve transected sciatic nerve regeneration. Iran J Biotechnol 15: 149-156, 2017.

26. Moldovan M, Pinchenko V, Dmytriyeva O, Pankratova S, Fugleholm K, Klingelhofer J, Bock E, Berezin V, Krarup C and Kiryushko D: Peptide mimetic of the S100A4 protein modulates peripheral nerve regeneration and attenuates the progression of neuropathy in myelin protein P0 null mice. Mol Med 19: 43-53, 2013.

27. Stratton JA, Holmes A, Rosin NL, Sinha S, Vohra M, Burma NE, Trang T, Midha R and Biernaskie J: Macrophages regulate schwann cell maturation after nerve injury. Cell Rep 24: 2561-2572.e6, 2018.
28. Kim KJ and Namgung U: Facilitating effects of Buyang Huanwu decoction on axonal regeneration after peripheral nerve transection. J Ethnopharmacol 213: 56-64, 2018.

29. Li R, Li DH, Zhang HY, Wang J, Li XK and Xiao J: Growth factors-based therapeutic strategies and their underlying signaling mechanisms for peripheral nerve regeneration. Acta Pharmacol Sin 41: 1289-1300, 2020.

30. El Seblani N, Welleford AS, Quintero JE, van Horne CG and Gerhardt GA: Invited review: Utilizing peripheral nerve regenerative elements to repair damage in the CNS. J Neurosci Methods 335: 108623, 2020.

31. Kuffler DP and Foy C: Restoration of neurological function following peripheral nerve trauma. Int J Mol Sci 21: 1808, 2020.

32. Alsmadi NZ, Bendale GS, Kanneganti A, Shihabeddin T, Nguyen AH, Hor E, Dash S, Johnston B, Granja-Vazquez R and Romero-Ortega MI: Glial-derived growth factor and pleiotrophin synergistically promote axonal regeneration in critical nerve injuries. Acta Biomater 78: 165-177, 2018.

33. Sweeney M and Foldes G: It takes two: Endothelial-perivascular cell cross-talk in vascular development and disease. Front Cardiovasc Med 5: 154, 2018.

34. Wang H, Zhu H, Guo Q, Qian T, Zhang P, Li S, Xue C and Gu X: Overlapping mechanisms of peripheral nerve regeneration and angiogenesis following sciatic nerve transection. Front Cell Neurosci 11: 323, 2017.

35. Chen G, Luo X, Wang W, Wang Y, Zhu F and Wang W: Interleukin- $1 \beta$ promotes schwann cells de-differentiation in wallerian degeneration via the c-JUN/AP-1 pathway. Front Cell Neurosci 13: 304, 2019.

36. Rhode SC, Beier JP and Ruhl T: Adipose tissue stem cells in peripheral nerve regeneration-in vitro and in vivo. J Neurosci Res 99: 545-560, 2021.

37. Manning BD and Toker A: AKT/PKB signaling: Navigating the network. Cell 169: 381-405, 2017.

(i) $\Theta$ This work is licensed under a Creative Commons Attribution-NonCommercial-NoDerivatives 4.0 International (CC BY-NC-ND 4.0) License. 